Das ergopraxis-Team (von links): Julia Belitz (Layout), Daniela Ottinger, Simone Gritsch, Maria Czyganowski, Annette Alkemade (Redaktion), Elke Baumann (Redaktionsleitung), Lioba Schlegel, Markus Böggemann (Marketing)

\title{
Frischer Wind!
}

_ Unsere Arbeitswelt befindet sich im Wandel. Der Akademisierungsprozess schreitet voran, Kostenträger und Mediziner fordern verstärkt Wirksamkeitsnachweise unserer Therapien. ergopraxis verfolgt die derzeitigen Entwicklungen für Sie, fasst aktuelle Forschungsergebnisse zusammen (॰ S. 12), stellt Ihnen ergotherapeutische Perspektiven vor und greift politische Themen auf. Wir haben uns Schutzhandschuhe angezogen und packen auch heiße Eisen wie zum Beispiel das Gehalt von Ergotherapeuten (๑ S. 8) für Sie an! In der zentralen Rubrik „Ergotherapie“ bieten wir Ihnen aktuelle Themen aus allen ergotherapeutischen Arbeitsfeldern.

_ In unserer täglichen Arbeit erleben wir häufig Kinder mit Problemen im Bereich der Koordination. Kanadische Ergotherapeuten setzen bei ihnen das sogenannte CO-OP ein - einen Ansatz, der in Deutschland immer bekannter wird. $\mathrm{Zu}$ Recht, wie ich finde, denn seine Wirksamkeit konnte schon in mehreren Studien belegt werden. Die Berliner Praxisinhaberin Angelika Oetken berichtet von ihren Erfahrungen mit dem CO-OP-Ansatz (๑S. 16).

_ Die Gesellschaft altert. Ergotherapeuten sind daher heute auch verstärkt im Bereich Geriatrie gefragt. Hier findet weitaus mehr statt als bloße Beschäftigungstherapie. Lesen Sie im Artikel von Corina Mohr, welche ergotherapeutischen Fördermöglichkeiten es für Menschen mit Demenz gibt (๑ S. 20).

_ Im Rahmen seiner Vortragsreise in Deutschland konnte ich den japanischen Professor Michael K. Iwama kennenlernen und für einen Artikel gewinnen. Er erläutert Ihnen anhand eines Fallbeispiels aus der Neurologie, wie Sie das von ihm mitentwickelte Kawa-Modell in der Praxis einsetzen können (๑ S. 24).

Viel Spaß beim Lesen der ergopraxis, die ab sofort monatlich erscheint, wünscht Ihnen Ihre

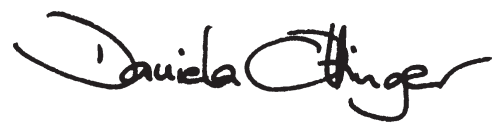

PS: Wie gefällt Ihnen die ergopraxis? Wir freuen uns über Ihre Meinung, Kommentare und Anregungen per E-Mail an ergopraxis@thieme.de!

\section{INTERNET}

Fachwissen für Ergotherapeuten

Sie interessieren sich für ergotherapeutische Fachbücher, Zeitschriften und Veranstaltungen? Dann schauen Sie doch mal im Internet unter www.thieme. de/ergoonline. Dort finden Sie zudem Assessments zum Downloaden und nützliche www-Tipps. 